\title{
¿Es el suicidio un evento prevenible?
}

\section{Is the suicide a preventable event?}

\author{
Adalberto Campo-Arias'; Yuly Suárez-Colorado²
}

Forma de citar: Campo-Arias A, Suárez-Colorado Y. Es el suicidio un evento prevenible. Rev Univ Ind Santander Salud. 2019; 51(3): 197-199. doi: http://dx.doi.org/10.18273/revsal.v51n3-2019002 (c) (1)

Con alto grado de certeza y basado en la evidencia disponible, muchos casos de suicidio se pueden prevenir. Pero, indiscutiblemente, es difícil predecir y prevenir todos los eventos ${ }^{1}$. Aunque, se pueden identificar los factores de riesgo biológicos, psicológicos, culturales, religiosos, sociales, económicos, políticos, etcétera² .

Las estrategias preventivas más frecuentes responden a los factores de riesgos; sin embargo, ningún factor en forma aislada o independiente, es capaz de predecir en sí mismo un caso de suicidio en particular ${ }^{2}$. El suicidio es un evento resultado de interacciones complejas de todas las variables antes anotadas y, por lo tanto, los casos de suicidio consumado no deberían generar sentimientos de culpa en familiares, amigos o profesionales de la salud ${ }^{3}$.

Se observan algunos avances en el conocimiento de las características, situaciones o predisponentes que llevan a las personas a la decisión del suicidio ${ }^{4}$. No obstante, se necesita más claridad y mayor comprensión global que explique todos los casos de suicidio. Aproximadamente, en el 10\% de los casos no se encuentran las causas o los factores predisponentes ${ }^{5}$.

La investigación científica intenta precisar los factores asociados según el patrón de escalonamientos de los comportamientos suicidas ${ }^{6}$. Aquellos que condicionan en particular la transición desde el deseo de muerte hasta el suicidio consumado, y los pasos intermedios como la ideación suicida, los planes y los intentos suicidas sin clara expectación de muerte ${ }^{6,7}$. Se necesita un modelo de predicción más satisfactorio, más allá de los factores de riesgo ${ }^{7}$. Los modelos estadísticos siempre basados en datos grupales son incapaces de hacer predicciones para casos singulares ${ }^{8,9}$. Dado las posibles combinaciones de factores para explicar y predecir los comportamientos suicidas, es necesario una mayor aproximación a la identificación de determinantes en cada individuo ${ }^{8}$.

La implementación de estrategias para la prevención del suicidio requiere de más investigación previa, debido a que se diseñan e implementan estrategias para la prevención no integrales ni integradas, sin la suficiente evidencia de efectividad ${ }^{10}$.

En la actualidad, las estrategias nacionales de prevención, donde tales existen, se limitan a medidas fragmentadas universales, selectivas o indicadas, como políticas de salud mental (no siempre llevadas a la práctica) o programas la reducción del consumo de alcohol y otras sustancias, acceso a la atención en salud mental, la restricción a métodos suicidas, la notificación responsable o difusión en medios de casos de suicidio, la concientización de la salud, las líneas telefónicas de ayuda, el seguimiento y apoyo comunitario y la evaluación y manejo de trastornos mentales, en particular los trastornos depresivos ${ }^{2}$.

De las estrategias antes anotadas, la evidencia indica que las acciones más efectivas son la restricción de métodos para suicidio, los programas escolares de prevención, el tratamiento farmacológico o psicoterapéutico para la depresión y el acceso a la atención en salud. Por el contrario, se necesitan más estudios para conocer la efectividad de

1. Universidad del Magdalena. Santa Marta, Colombia.

2. Universidad Cooperativa de Colombia. Santa Marta, Colombia.

Correspondencia: Yuly Suárez-Colorado. Dirección: carretera troncal del Caribe sector Mamatoco. Santa Marta, Colombia. Correo electrónico: ysuc91@gmail.com. Teléfono: (5) 4209604 Ext. 5580 
la identificación de personas en alto riesgo de suicidio en los centros médicos de atención primaria, la educación pública general, las pautas para la información de casos en medios de comunicación, la capacitación de los guardianes, la educación de los médicos y los servicios de asistencia telefónica e Internet ${ }^{10}$.

La prevención del suicidio en tiempo real es un reto direccionado por el desarrollo tecnológico y la innovación. La marcha de proyectos sobre dispositivos electrónicos para el monitoreo del riesgo de suicidio, a través de factores como la conducción eléctrica de la piel, la agitación motora y el ritmo cardiaco, requieren de mayor evidencia; además, se deben resolver los posibles problemas éticos implícitos en la predicción del suicidio con el uso de estas nuevas tecnologías ${ }^{11}$.

Las estrategias efectivas e innovadoras son necesarias; pero, no son suficientes sin una perspectiva sindémica del suicidio. El término sindemia se refiere a la presentación de dos o más enfermedades o condiciones en una misma persona y están estrechamente relacionadas con las desigualdades sociales y económicas persistentes en las que vive. La sindemia permite reconocer, como la violencia estructural y las interacciones fundamentadas en el estigma, la discriminación y exclusión, conforman un sistema de vulnerabilidad generador de sufrimiento y problemas que pueden motivar el suicidio ${ }^{12}$.

Al respecto, el $27 \%$ de la población colombiana informa necesidades básicas insatisfechas ${ }^{13}$. Colombia es uno de los países con mayor desigualdad en el contexto mundial con un coeficiente de desigualdad de Gini de $0,53^{14}$. Asimismo, entre el $12,1 \%$ y $21,3 \%$ de la población colombiana en edades entre 12 y 60 años se percibe discriminada y rechazada ${ }^{15}$. En población colombiana se evidenció una asociación entre determinantes estructurales, como desigualdad económica, y la tasa de intentos de suicidio ${ }^{16}$; la desigualdad del ingreso y el desempleo se encuentran relacionados con el mismo ${ }^{17}$.

La antropología médica concibe a la sindemia como una alternativa para comprender el proceso salud-enfermedadatención desde una visión sociocultural; ésta supera algunas limitaciones del modelo biomédico, al considerar la enfermedad como una entidad discreta ${ }^{18}$. Esta perspectiva es importante en los modelos explicativos de enfermedades no trasmisibles como el suicidio. Una aproximación sindémica del suicidio estimaría mejor las interrelaciones entre las enfermedades, condiciones y relaciones sociales nocivas; además de considerar como la estructura social configura el proceso salud-enfermedad-atención. El enfoque sindémico del suicidio lo concibe como una forma de sufrimiento social ${ }^{12}$.

El suicidio es todo un reto, un fenómeno difícil de comprender desde la perspectiva clásica de la epidemiología, se necesita una aproximación sindémica para la comprensión del suicidio y, en consecuencia, estrategias y acciones integradas e integrales de prevención. El estudio del suicidio demanda de una aproximación interdiscipilinar y multidisciplinar desde la investigación en psicología, enfermería, medicina, sociología y antropología, entre otras. Se necesita un proceso planeado de investigación para responder con absoluta certeza ¿es el suicidio un evento prevenible?

\section{Referencias}

1. Arafat SMY. Not all suicide is preventable: a letter to the editor. Asian J Psychiatry. 2018; 36(1): 19. doi: https://doi.org/10.1016/j.ajp.2018.06.003.

2. Organización Mundial de la Salud. Prevención del Suicidio. Un Imperativo Global. 2014: 1-89.

3. Pridmore S, Pridmore W. Not all suicide is preventable. Asian J Psychiatry. 2018; 36 (1): 8-9. doi: https://doi.org/10.1016/j.ajp.2018.05.027

4. Mann J, Apter A, Bertolote J, Beautrais A, Currier D, Haas A, et al. Suicide prevention strategies: a systematic review. JAMA. 2005; 294(16): 2064-2074. doi: https://doi.org/10.1001/jama.294.16.2064.

5. Schwartz-Lifshitz M, Zalsman G, Giner L, Oquendo MA. Can we really prevent suicide? Cur Psychiatry Report. 2012; 14(6): 624-633. doi: https://doi.org/10.1007/s11920-012-0318-3.

6. Klonsky E, May A, Saffer B. Suicide, suicide attempts, and suicidal ideation. An Rev Clin Psychol. 2016; 12: 307-330. doi: https://doi.org/10.1146/annurev-clinpsy-021815-093204.

7. Klonsky E, Saffer B, Bryan C. Ideation-to-action theories of suicide: a conceptual and empirical update. Cur Op 
Psychol. 2018; 22(1): 38-43. doi: https://doi.org/10.1016/j.copsyc.2017.07.020.

8. Fisher AJ, Medaglia JD, Jeronimus BF. Lack of group-to-individual generalizability is a threat to human subjects research. Proc Natl Acad Sci. 2018; 115(27): E6106-115. doi: https://doi.org/10.1073/pnas.1711978115.

9. Barroso AA. Alcances y limitaciones de un enfoque de riesgos para comprender el suicidio. Rev Hosp Psiquiatr Habana. 2017; 14(1): 7.

10. Zalsman G, Hawton K, Wasserman D, Van Heeringen K, Arensman E, Sarchiapone M, et al. Suicide prevention strategies revisited: 10-year systematic review. Lancet Psychiatry. 2016; 3(7): 646-659. doi: https://doi.org/10.1016/S2215-0366(16)30030-X.

11. Bentley K, Kleiman E, Elliott G, Huffman J, Nock M. Real-time monitoring technology in single-case experimental design research: opportunities and challenges. Behav Res Ther. 2018; (ahead of print). doi: https://doi.org/10.1016/j.brat.2018.11.017

12. Singles M, Claire S. Syndemics and public health: Reconceptualizing disease in bio-social context. Med Anthropol Q. 2003; 17 (4): 423-441.

13. Departamento Administrativo Nacional de Estadística. Bolerines Censo. 2005.

14. Banco Mundial. Sobre la pobreza y la prosperidad compartida: abordar la desigualdad. 2016.

15. Minsalud-Colciencias. Encuesta Nacional de Salud Mental, 2015. Bogotá: Javegraf; 2015.

16. Arenas A, Gómez-Restrepo C, Rondón M. Factores asociados a la conducta suicida en Colombia. Resultados de la Encuesta Nacional de Salud Mental 2015. Rev Colomb Psiquiatr. 2016; 45(1): 68-75.

17. Dávila-Cervantes C, Pardo-Montaño A. Impacto de factores socioeconómicos en la mortalidad por suicidios en Colombia, 2000-2013. Rev Ger Polit Salud. 2017; 16(33): 36-51. doi: https://doi.org/10.11144/ Javeriana.rgps16-33.ifsm.

18. Mendenhall E, Kohrt B, Norris S, Ndetei D, Prabhakaran D. Non-communicable disease syndemics: poverty, depression, and diabetes among low-income populations. Lancet. 2017; 389(10072): 951-963. doi: https://doi.org/10.1016/S0140-6736(17)30402-6. 